\title{
1,n-Bis(phenylsulfanyl)alkanes as precursors of 1,n-dilithio compounds: synthesis of diols
}

\author{
Abdeslam Abou, Francisco Foubelo, and Miguel Yus* \\ Departamento de Química Orgánica and Instituto de Síntesis Orgánica (ISO), Facultad de \\ Ciencias, Universidad de Alicante, Apartado 99, E-03080 Alicante, Spain \\ E-mail:yus@ua.es
}

This paper is dedicated to Professor Joan Bosch on occasion of his $60^{\text {th }}$ anniversary

\begin{abstract}
The reaction of 1,n-bis(phenylsulfanyl)alkanes $1(n=2-6)$ with an excess of lithium powder and a catalytic amount of 4,4'-di-tert-butylbiphenyl (DTBB; $2.5 \mathrm{~mol} \%$ ) in the presence of a carbonyl compound [PhCHO, Et ${ }_{2} \mathrm{CO}$; Barbier conditions], in $\mathrm{THF}$ at $-78{ }^{\circ} \mathrm{C}$ leads, after hydrolysis with water, to the expected 1, $(n+2)$-diols 6 , yields being $<19 \%$ for $n=2$ and in the range of $31-78 \%$ for $n=3-6$. When the lithiation is performed in a two-step process (Grignard conditions), first with the same lithiating reagent and then with different carbonyl compounds $\left[\mathrm{Bu}^{t} \mathrm{CHO}, \mathrm{PhCHO}\right.$, $\mathrm{PhCH}_{2} \mathrm{CHO}, \mathrm{Et}_{2} \mathrm{CO}$, $\left(\mathrm{CH}_{2}\right)_{5} \mathrm{CO},\left(\mathrm{CH}_{2}\right)_{7} \mathrm{CO}$, (-)-menthone, $\mathrm{Ph}_{2} \mathrm{CO}$, diols 6 are obtained in significant lower yields except for 1,3-bis(phenylsulfanyl)propane $\mathbf{1}(\mathrm{n}=3)$.
\end{abstract}

Keywords. DTBB-catalyzed lithiation, lithium-naphthalene, sulfur-lithium exchange, electrophilic substitution, diols

\section{Introduction}

Phenylsulfanylalkanes I have been used extensively as precursors of organolithium compounds by applying two different methodologies. One is the well-known $\alpha$-deprotonation ${ }^{1}$ by using an appropriate organolithium compound as a base (Scheme 1). A sulfur stabilized carbanion species II is obtained due to the effect of the sulfur atom on adjacent carbanions by electron backdonation into vacant d-orbitals. ${ }^{2}$ Through this methodology dithioacetals, and among them 1,3dithiane, have been widely used as precursors of organolithium compounds acting as masked nucleophilic acylating agents. ${ }^{3}$ The other methodology consists in the use of lithium metal either alone or in the presence of a stoichiometric ${ }^{4}$ or catalytic ${ }^{5}$ amount of an arene as the lithiation reagent, a sulfur-lithium exchange taking place in the process to generate the corresponding organolithium compound III (Scheme 1). On the other hand, dilithium compounds ${ }^{6}$ are 
interesting intermediates in synthetic organic chemistry because, by reaction with two electrophiles, polyfunctionalized molecules can be obtained in only one step. Halogen-lithium exchange $^{7}$ is the most commonly used method to prepare organolithium compounds, however only in some cases $(1, \mathrm{n} \text {-dihaloalkanes when } \mathrm{n}>5)^{8}$ and under special reaction conditions (low temperatures and lithiation in the presence of the electrophile) dihalocompounds can be used as precursors of the corresponding dilithium derivatives. This originates from the fact that after the first lithiation, the resulting intermediate decomposes due to the lack of compatibility of the carbon-lithium bond initially formed and the remaining carbon-halogen bond. In this paper we study the use of $1, n$-bis(phenylsulfanyl)alkanes $\mathbf{1}$ as precursors of $1, n$-dilithium intermediates ${ }^{9}$ using an excess of lithium and a catalytic amount of 4,4'-di-tert-butylbiphenyl (DTBB) as the lithiation reagent and explore the applicability of this methodology to the synthesis of diols.

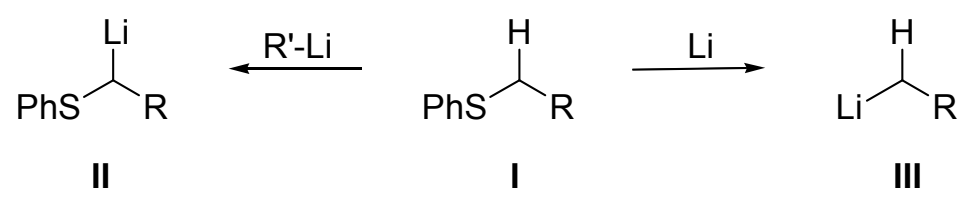

Scheme 1. Lithiation of phenylsulfanylalkanes

\section{Results and Discussion}

The reaction of different 1,n-bis(phenylsulfanyl)alkanes $\mathbf{1}$ (easily prepared from the corresponding 1,n-dibromoalkanes by reaction with sodium thiophenolate in methanol at room temperature) with an excess of lithium (1:10 molar ratio; theoretically 1:4 molar ratio) and a catalytic amount of DTBB (1:0.1 molar ratio, $2.5 \mathrm{~mol} \%)$ in the presence of different carbonyl compounds (1:3 molar ratio) in THF at $-78^{\circ} \mathrm{C}$ for $c a .3 \mathrm{~h}$, followed by hydrolysis with water at temperatures ranging between $-78{ }^{\circ} \mathrm{C}$ and room temperature (Method $\mathrm{A}$ ), led to the expected diols 6 (Scheme 2 and Table 1, entries 1,3, 10-12).

Concerning the mechanistic pathway for the reaction shown in Scheme 2, we think that after the first lithiation, the phenylsulfanyl-lithio intermediate $\mathbf{2}$ reacts with the electrophile present in the reaction medium to give the phenylsulfanyl-alkoxide $\mathbf{3}$, which undergoes a new sulfur-lithium exchange to afford the oxygen-functionalized organolithium compound 4, which in the presence of the electrophile gives the corresponding dialkoxide 5, precursor of the diol 6 by final hydrolysis (Scheme 2). Yields are low for compounds 6 with $n=2,4$ and 5 (Table 1, entries 1, 10 and 11) and relatively high for compounds 6 with $\mathrm{n}=3$ and 6 (Table 1, entries 2 and 12). These results could be explained considering the stability of the corresponding initially formed phenylsulfanyl-lithio intermediates 2 (Chart 1). Thus, compound 2 with $n=2$ is very unstable and is prone to undergo $\beta$-elimination to give ethylene. ${ }^{4 a, b}$ However, its homologous compound 2 with $n=3$ is much more stable, probably due to an intramolecular coordination of the metal with the sulfur atom ${ }^{10}$ avoiding in this way the elimination process to yield 
cyclopropane. Surprisingly intermediates 2 with $n=4$ and 5 seem to be less stable than that with $\mathrm{n}=3$, intramolecular proton abstraction being a possible decomposition pathway for these systems, due to the acidity of the hydrogen atoms at the carbon bonded to the sulfur atom. Finally, intermediate $\mathbf{2}$ with $\mathrm{n}=6$ is quite stable and behaves as a normal organolithium compound.
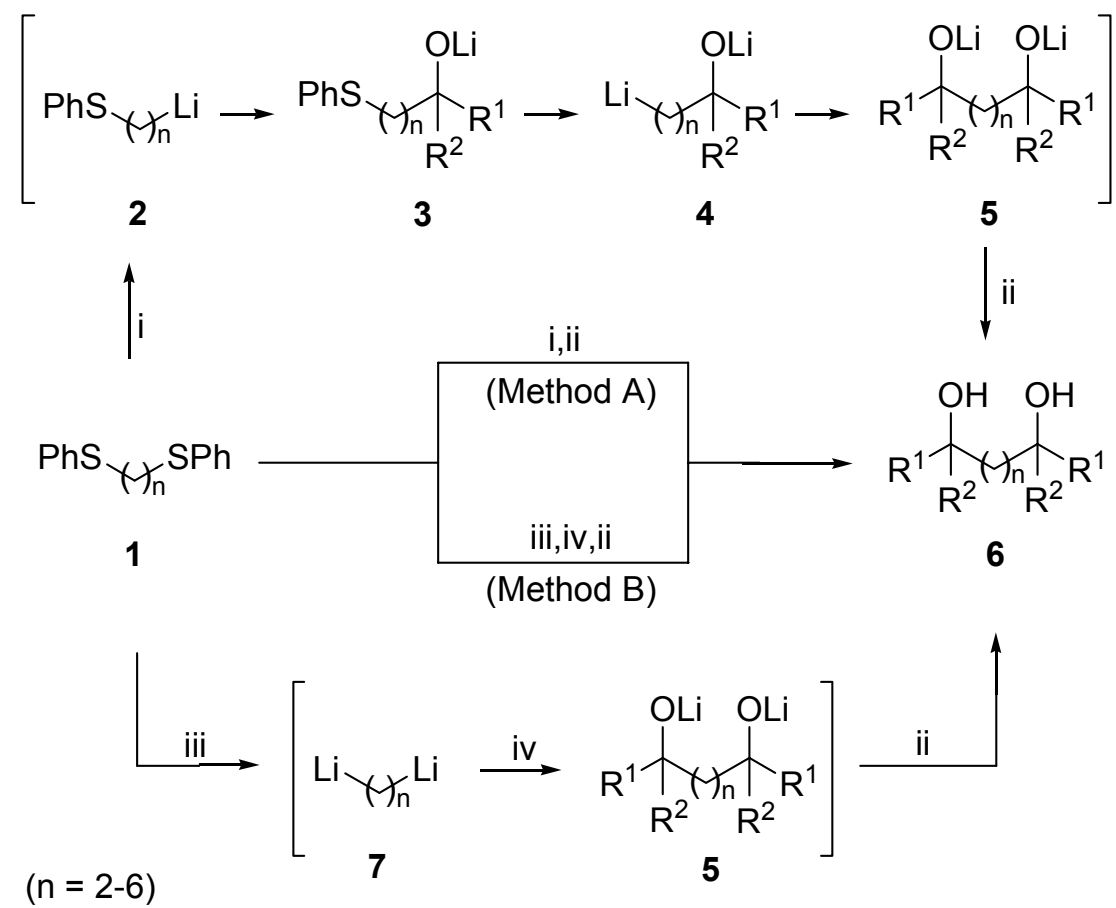

Scheme 2. Reagents and conditions: (i) Li, DTBB (2.5 mol \%), $\mathrm{R}^{1} \mathrm{R}^{2} \mathrm{CO}, \mathrm{THF},-78^{\circ} \mathrm{C}$; (ii) $\mathrm{H}_{2} \mathrm{O}$, -78 to $20^{\circ} \mathrm{C}$; (iii) $\mathrm{Li}$, DTBB (2.5 mol \%), THF, $-78^{\circ} \mathrm{C}$; (iv) $\mathrm{R}^{1} \mathrm{R}^{2} \mathrm{CO},-78^{\circ} \mathrm{C}$.
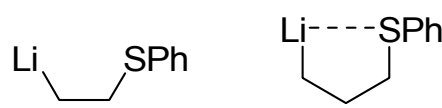

$2(n=2)$

$2(n=3)$

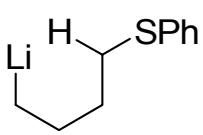

$2(n=4)$

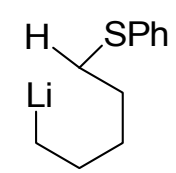

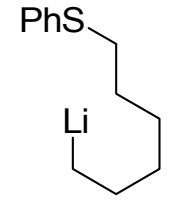

$2(n=6)$

Chart 1. Organolithium intermediates 2 
Table 1. Double lithiation of 1,n-bis(phenylsulfanyl)alkanes 1. Preparation of diols 6

\begin{tabular}{|c|c|c|c|c|c|c|}
\hline \multirow[b]{3}{*}{ Entry } & \multirow[b]{3}{*}{$\mathrm{n}$} & \multirow[b]{3}{*}{ Electrophile } & \multicolumn{4}{|c|}{ Product $^{\mathrm{a}}$} \\
\hline & & & \multirow[b]{2}{*}{ No. } & \multirow[b]{2}{*}{ Structure } & \multicolumn{2}{|l|}{ Yield $(\%)^{b}$} \\
\hline & & & & & Method A & Method B \\
\hline 1 & 2 & $\mathrm{Et}_{2} \mathrm{CO}$ & $6 \mathbf{a}$ & & 19 & 0 \\
\hline 2 & 3 & $\mathrm{Bu}^{t} \mathrm{CHO}$ & $6 b$ & & -- & $62^{c}$ \\
\hline 3 & 3 & $\mathrm{PhCHO}$ & $6 c$ & & $71^{\mathrm{c}}$ & $69^{c}$ \\
\hline 4 & 3 & $\mathrm{PhCH}_{2} \mathrm{CHO}$ & 6d & & -- & $35^{\mathrm{c}}$ \\
\hline 5 & 3 & $\mathrm{Et}_{2} \mathrm{CO}$ & $6 e$ & & -- & 59 \\
\hline 6 & 3 & $\left(\mathrm{CH}_{2}\right)_{5} \mathrm{CO}$ & $6 f$ & & -- & 66 \\
\hline 7 & 3 & $\left(\mathrm{CH}_{2}\right)_{7} \mathrm{CO}$ & $6 \mathrm{~g}$ & & -- & 68 \\
\hline 8 & 3 & (-)-menthone & $6 h$ & & -- & $58^{\mathrm{d}}$ \\
\hline 9 & 3 & $\mathrm{Ph}_{2} \mathrm{CO}$ & $6 \mathbf{i}$ & & -- & 45 \\
\hline 10 & 4 & $\mathrm{Et}_{2} \mathrm{CO}$ & $6 \mathbf{j}$ & & 31 & 9 \\
\hline 11 & 5 & $\mathrm{Et}_{2} \mathrm{CO}$ & $6 \mathbf{k}$ & & 36 & 26 \\
\hline 12 & 6 & $\mathrm{PhCHO}$ & 61 & & 78 & 65 \\
\hline
\end{tabular}

${ }^{\text {a }}$ All products 5 were $>95 \%$ pure (GLC and/or $300 \mathrm{MHz}{ }^{1} \mathrm{H}$ NMR) and were fully characterised by spectroscopic means (IR, ${ }^{1} \mathrm{H}$ and ${ }^{13} \mathrm{C} \mathrm{NMR}$, and LR and HR mass spectrometry).

${ }^{\mathrm{b}}$ Isolated yields of compounds $\mathbf{5}$ after column chromatography (silica gel, hexane/ethyl acetate).

${ }^{\mathrm{c}}$ Obtained as a ca. 1:1 mixture of diastereomers (NMR).

${ }^{d}$ The diastereomer shown in this table was exclusively obtained (see Text). 
We also studied the lithiation of compounds 1 in the absence of the electrophile (Grignardtype conditions). Thus, the reaction of compounds 1 with an excess of lithium (1:10 molar ratio; theoretically 1:4 molar ratio) and a catalytic amount of DTBB (1:0.1 molar ratio, $2.5 \mathrm{~mol} \%)$ in THF at $-78^{\circ} \mathrm{C}$ for $1 \mathrm{~h}$, followed by the addition of 2.2 equivalents of a carbonyl compound at the same temperature and final hydrolysis with water at temperatures ranging between $-78{ }^{\circ} \mathrm{C}$ and room temperature (Method B), led to the expected diols 6 (Scheme 2 and Table 1).

In the case of 1,2-bis(phenylsulfanyl)ethane $(\mathbf{1}, \mathrm{n}=2$, Table 1 , entry 1$)$, intermediate $\mathbf{2}(\mathrm{n}=$ 2) decomposed rapidly before a second lithiation could take place. So after addition of 3pentanone as electrophile, nothing of the expected diol 6a was isolated nor detected by tandem GC/MS analysis. That indicates, as previously commented, that intermediates of type $\mathbf{2}(\mathrm{n}=2)$ show a high tendency to undergo elimination. ${ }^{4 a, b}$ Such elimination takes place almost exclusively (Scheme 2, Table 1, entry 1) even in the presence of the electrophile (Barbier-type conditions, Method A), which probably explains the low yield. A very good yield was obtained (similar under Barbier-reaction conditions) in the case of 1,3-bis(phenylsulfanyl)propane $(\mathbf{1}, \mathrm{n}=3)$ (Table 1, entry 3), which is a proof that intermediate 2 with $\mathrm{n}=3$ was a stable species. Surprisingly, for 1,4- and 1,5-bis(phenylsulfanyl)alkanes 1, after lithiation and reaction with 3pentanone, the diols $\mathbf{6 j}$ and $\mathbf{6 k}$ were obtained in only 9 and $26 \%$ yield (Table 1 , entries 10 and 11), respectively. For these starting materials, method B provides lower yields than method A. Finally, a slightly lower yield was obtained from 1,6-bis(phenylsulfanyl)hexane $(2, n=6)$ after lithiation followed by reaction with benzaldehyde (Method B) compared to lithiation in the presence of the electrophile (Method A, Table 1, entry 12).

The best yields of diols 6 were obtained in general under Barbier-type reaction conditions (Method A) but for 1,5-diols 6 with $\mathrm{n}=3$, the Grignard-type reaction conditions (Method B) led to similar yields. Thus, the lithiation of 1,3 -bis(phenylsulfanyl)propane $(\mathbf{1}, \mathrm{n}=3)$ followed by reaction with different carbonyl compounds as electrophiles under the reaction conditions described for Method B led to the corresponding 1,5-diols 6 in good yields (Scheme 2, Table 1, entries 2, 4-9). When a prochiral carbonyl compound was used as electrophile, a ca. 1:1 diastereomeric mixture of the corresponding diols was obtained (Table 1, entries 2-4), a single diastereoisomer being obtained from (-)-menthone, the one resulting for the attack of the organolithium compound to the less hindered face of the carbonyl group (Table 1, entry 8).

In conclusion, we report here the lithiation of 1,n-bis(phenylsulfanyl)alkanes under DTBBpromoted conditions, which allows the preparation of symmetrically substituted diols $\mathbf{6}$ by using carbonyl compounds as electrophiles. The process can be performed in a single synthetic operation (lithiation in the presence of the electrophile; Barbier-type conditions) or in a two-step process (lithiation followed by addition of the electrophile: Grignard-type conditions). The yields of these processes depend strongly on the stability of the functionalized organolithium intermediates, 1,5- and 1,8-diols being easily accessible in moderate to good yields by employing this methodology. 


\section{Experimental Section}

General Procedures. All reactions were carried out under an atmosphere of argon in oven-dried glassware. All reagents were commercially available (Acros, Aldrich) and were used without further purification. Commercially available anhydrous THF $(99.9 \%$, water content $\leq 0.006 \%$, Acros) was used as solvent in all the lithiation reactions. IR spectra were measured (film) with a Nicolet Impact 400 D-FT Spectrometer. NMR spectra were recorded with a Bruker AC-300 or a Bruker ADVANCE DRX-500 using $\mathrm{CDCl}_{3}$ as the solvent. LRMS and HRMS were measured with Shimadzu GC/HS QP-5000 and Finingan MAT95 S spectrometers, respectively. The purity of volatile products and the chromatographic analyses (GLC) were determined with a flame ionisation detector and a $12 \mathrm{~m}$ capillary column ( $0.2 \mathrm{~mm}$ diam., $0.33 \mu \mathrm{m}$ film thickness), using nitrogen $(2 \mathrm{~mL} / \mathrm{min})$ as carrier gas, $\mathrm{T}_{\text {injector }}=275^{\circ} \mathrm{C}, \mathrm{T}_{\text {detector }}=300^{\circ} \mathrm{C}, \mathrm{T}_{\text {column }}=60^{\circ} \mathrm{C}(3 \mathrm{~min})$ and $60-270^{\circ} \mathrm{C}\left(15^{\circ} \mathrm{C} / \mathrm{min}\right), \mathrm{P}=40 \mathrm{kPa}$. Specific rotations were determined with a PerkinElmer 341 digital polarimeter.

\section{Preparation of 1,n-bis(phenylsulfanyl)alkanes (1)}

Isolation of compounds 1. General procedure. Thiophenol (2.42 g, $21.0 \mathrm{mmol})$ was added to a solution of $\mathrm{KOH}(1.25 \mathrm{~g}, 22.5 \mathrm{mmol})$ in methanol $(40 \mathrm{~mL})$ at $0{ }^{\circ} \mathrm{C}$. After $10 \mathrm{~min}$, the corresponding 1,n-dibromoalkane $(10.0 \mathrm{mmol})$ was added, and stirring was continued at $20{ }^{\circ} \mathrm{C}$ for $4 \mathrm{~h}$. Then, the solvent was removed in a rotary evaporator, and the residue was hydrolyzed with water and extracted with ethyl acetate. The organic layer was dried over anhydrous magnesium sulfate and evaporated (15 Torr). The residue was purified by column chromatography (silica gel; hexane) to yield pure products $\mathbf{1}$. Yields and physical and spectroscopic data as well as literature references follow.

1,2-Bis(phenylsulfanyl)ethane $(\mathbf{1}, \mathbf{n}=\mathbf{2}) .{ }^{11}$ White solid; mp $70-71{ }^{\circ} \mathrm{C}$ (hexane), $96 \%$ yield; $R_{\mathrm{f}}$ 0.32 (hexane); $v(\mathrm{KBr}) 3080,3060 \mathrm{~cm}^{-1}(\mathrm{ArH}) ; \delta_{\mathrm{H}} 3.08\left(4 \mathrm{H}, \mathrm{s}, \mathrm{CH}_{2} \mathrm{CH}_{2}\right), 7.18-7.31(10 \mathrm{H}, \mathrm{m}$, $\mathrm{ArH}) ; \delta_{\mathrm{C}} 33.3\left(\mathrm{CH}_{2}\right), 126.5,129.0,130.0,134.95(\mathrm{ArC}) ; m / z 246\left(\mathrm{M}^{+}, 30 \%\right), 137$ (100), 135 (21), 109 (48), 65 (10).

1,3-Bis(phenylsulfanyl)propane $(\mathbf{1}, \mathbf{n}=\mathbf{3}) .{ }^{12}$ Colourless oil, $91 \%$ yield; $R_{\mathrm{f}} 0.25$ (hexane); $v$ (film) $3072,3054 \mathrm{~cm}^{-1}(\mathrm{ArH}) ; \delta_{\mathrm{H}} 1.87\left(2 \mathrm{H}\right.$, quintet, $\left.J=7.3 \mathrm{~Hz}, \mathrm{CH}_{2} \mathrm{CH}_{2} \mathrm{CH}_{2}\right), 2.95(4 \mathrm{H}, \mathrm{t}, J=$ $\left.7.3 \mathrm{~Hz}, 2 \times \mathrm{PhSCH}_{2}\right), 7.09-7.27(10 \mathrm{H}, \mathrm{m}, \mathrm{ArH}) ; \delta_{\mathrm{C}} 27.9,31.9\left(\mathrm{CH}_{2}\right), 125.7,128.6,128.9,135.8$ (ArC); m/z 260 (M , 100\%), 151 (38), 149 (17), 135 (25), 123 (36), 117 (41), 109 (28), 77 (12).

1,4-Bis(phenylsulfanyl)butane $(\mathbf{1}, \mathbf{n}=4) .{ }^{13}$ White solid; mp $58-59{ }^{\circ} \mathrm{C}$ (hexane), $82 \%$ yield; $R_{\mathrm{f}}$ 0.27 (hexane); $v(\mathrm{KBr}) 3074,3054 \mathrm{~cm}^{-1}(\mathrm{ArH}) ; \delta_{\mathrm{H}} 1.76-1.77\left[4 \mathrm{H}, \mathrm{m}, \mathrm{CH}_{2}\left(\mathrm{CH}_{2}\right)_{2} \mathrm{CH}_{2}\right], 2.90-2.94$ $\left(4 \mathrm{H}, \mathrm{m}, 2 \times \mathrm{PhSCH}_{2}\right), 7.15-7.32(10 \mathrm{H}, \mathrm{m}, \mathrm{ArH}) ; \delta_{\mathrm{C}} 28.0,33.2\left(\mathrm{CH}_{2}\right), 125.9,128.8,129.2,136.4$ (ArC); m/z $274\left(\mathrm{M}^{+}, 30 \%\right), 165$ (100), 123 (49), 109 (16).

1,5-Bis(phenylsulfanyl)pentane $(\mathbf{1}, \mathbf{n}=\mathbf{5}) .{ }^{14}$ Colourless oil, $90 \%$ yield; $R_{\mathrm{f}} 0.30$ (hexane); $v$ (film) $3074,3054 \mathrm{~cm}^{-1}(\mathrm{ArH}) ; \delta_{\mathrm{H}} 1.51-1.61\left[6 \mathrm{H}, \mathrm{m}, \mathrm{CH}_{2}\left(\mathrm{CH}_{2}\right)_{3} \mathrm{CH}_{2}\right], 2.84(4 \mathrm{H}, \mathrm{t}, J=7.0 \mathrm{~Hz}$, $\left.2 \times \mathrm{PhSCH}_{2}\right), 7.10-7.28(10 \mathrm{H}, \mathrm{m}, \mathrm{ArH}) ; \delta_{\mathrm{C}} 27.6,28.4,33.1\left(\mathrm{CH}_{2}\right), 125.5,128.6,128.7,136.5$ (ArC); $m / z 288\left(\mathrm{M}^{+}, 61 \%\right), 179$ (100), 178 (21), 123 (76), 110 (31), 109 (25), 69 (24). 
1,6-Bis(phenylsulfanyl)hexane $(\mathbf{1}, \mathbf{n}=\mathbf{6}) .{ }^{15}$ White solid; mp $80-81{ }^{\circ} \mathrm{C}$ (hexane), $85 \%$ yield; $R_{\mathrm{f}}$ 0.28 (hexane); $v(\mathrm{KBr}) 3071,3054 \mathrm{~cm}^{-1}(\mathrm{ArH}) ; \delta_{\mathrm{H}} 1.40-1.47\left(4 \mathrm{H}, \mathrm{m}, J=7.3 \mathrm{~Hz}, 2 \times \mathrm{CH}_{2}\right), 1.59-$ $1.68\left(4 \mathrm{H}, \mathrm{m}, 2 \times \mathrm{CH}_{2}\right), 2.84\left(4 \mathrm{H}, \mathrm{t}, J=7.0 \mathrm{~Hz}, 2 \times \mathrm{PhSCH}_{2}\right), 2.85-2.94\left(4 \mathrm{H}, \mathrm{m}, 2 \times \mathrm{PhSCH}_{2}\right), 7.15-$ $7.32(10 \mathrm{H}, \mathrm{m}, \mathrm{ArH}) ; \delta_{\mathrm{C}} 28.3,28.9,33.4\left(\mathrm{CH}_{2}\right), 125.7,128.8,128.9,136.8(\mathrm{ArC}) ; \mathrm{m} / z 302\left(\mathrm{M}^{+}\right.$, 100\%), 193 (61), 192 (21), 123 (78), 110 (38), 109 (30), 83 (16), 82 (21), 55 (27).

Double lithiation of compounds 1 in the presence of a carbonyl compound as electrophile (Barbier-type reaction conditions, Method A). Preparation of diols 6

Isolation of compound 6. Method A: General procedure. To a blue suspension of lithium powder $(0.070 \mathrm{~g}, 10 \mathrm{mmol})$ and a catalytic amount of DTBB $(0.027 \mathrm{~g}, 0.1 \mathrm{mmol})$ in THF $(3 \mathrm{~mL})$ was slowly added a solution of the corresponding 1,n-bis(phenylsulfanyl)alkane 1 (1.0 mmol) and the corresponding carbonyl compound $\left(\mathrm{R}^{1} \mathrm{R}^{2} \mathrm{CO}, 3.0 \mathrm{mmol}\right)$ in $\mathrm{THF}(1.2 \mathrm{~mL})$ during ca. $3 \mathrm{~h}$ at $-78{ }^{\circ} \mathrm{C}$. After the addition, the reaction mixture was stirred for $15 \mathrm{~min}$ at the same temperature. Then, it was hydrolyzed with water $(4 \mathrm{~mL})$ and extracted with ethyl acetate $(3 \times 10 \mathrm{~mL})$. The organic layer was dried over anhydrous magnesium sulfate and evaporated (15 Torr). The residue was purified by column chromatography (silica gel; hexane/ethyl acetate) to yield pure products 5. Yields and structures are included in Table 1. Physical and spectroscopic data as well as literature references follow.

3,6-Diethyloctane-3,6-diol (6a). ${ }^{16}$ Colourless oil; $R_{\mathrm{f}} 0.21$ (hexane/ethyl acetate: $2 / 1$ ); $v$ (film) $3520-3190(\mathrm{OH}), 2972,2935,2880,1460 \mathrm{~cm}^{-1} ; \delta_{\mathrm{H}} 0.86\left(12 \mathrm{H}, \mathrm{t}, J=7.5 \mathrm{~Hz}, 4 \times \mathrm{CH}_{3}\right), 1.45[4 \mathrm{H}, \mathrm{s}$, $\left.\left(\mathrm{CH}_{2}\right)_{2}\right], 1.48\left[8 \mathrm{H}, \mathrm{q}, J=7.5 \mathrm{~Hz}, 2 \times \mathrm{CH}_{3} \mathrm{CH}_{2}\right), 1.75(2 \mathrm{H}$, br s, $2 \times \mathrm{OH}) ; \delta_{\mathrm{C}} 7.8\left(\mathrm{CH}_{3}\right), 30.9,31.3$ $\left(\mathrm{CH}_{2}\right), 74.4(\mathrm{COH}) ; \mathrm{m} / z 166\left(\mathrm{M}^{+}-2 \mathrm{H}_{2} \mathrm{O}, 1 \%\right), 156$ (11), 155 (100), 137 (52), 98 (33), 95 (18), 87 (56), 83 (26), 69 (31), 57 (91), 55 (30); HRMS: $\mathrm{M}^{+}-\mathrm{H}_{2} \mathrm{O}$, found 184.1833. $\mathrm{C}_{12} \mathrm{H}_{24} \mathrm{O}$ requires 184.1827.

1,5-Diphenylpentane-1,5-diol (6c). ${ }^{17}$ Diastereomeric mixture: Colourless oil; $R_{\mathrm{f}} 0.19$ (hexane/ethyl acetate: 2/1); $v$ (film) 3530-3180 (OH), 3062, 3029, 2980, 2938, 2865, $1454 \mathrm{~cm}^{-1}$; $\delta_{\mathrm{H}} 1.43\left(2 \mathrm{H}\right.$, quintet, $\left.J=7.9 \mathrm{~Hz}, \mathrm{CH}_{2} \mathrm{CH}_{2} \mathrm{CH}_{2}\right), 1.52-1.81\left(4 \mathrm{H}, \mathrm{m}, 2 \times \mathrm{CH}_{2} \mathrm{CH}\right), 2.56(2 \mathrm{H}$, br s, $2 \times \mathrm{OH}), 4.58(2 \mathrm{H}, \mathrm{t}, J=7.3 \mathrm{~Hz}, 2 \times \mathrm{CHOH}), 7.23-7.33(10 \mathrm{H}, \mathrm{m}, \mathrm{ArH}) ; \delta_{\mathrm{C}} 22.0,22.1,38.6,38.7$ $\left(\mathrm{CH}_{2}\right), 74.1,74.3(\mathrm{CHOH}), 125.8,127.4,128.3,144.7(\mathrm{ArC}) ; m / z 238\left(\mathrm{M}^{+}-\mathrm{H}_{2} \mathrm{O}, 4 \%\right), 129$ (22), 105 (20), 104 (100), 91 (18), 77 (21).

3,8-Diethyldecane-3,8-diol (6j). ${ }^{18}$ White solid; mp 72-73 ${ }^{\circ} \mathrm{C}$ (dichloromethane/hexane); $R_{\mathrm{f}} 0.25$ (hexane/ethyl acetate: 2/1); $v(\mathrm{KBr}) 3520-3230(\mathrm{OH}), 2963,2943,2878,1461 \mathrm{~cm}^{-1} ; \delta_{\mathrm{H}} 0.85$ $\left(12 \mathrm{H}, \mathrm{t}, J=7.5 \mathrm{~Hz}, 4 \times \mathrm{CH}_{3}\right), 1.27-1.40\left[10 \mathrm{H}, \mathrm{m},\left(\mathrm{CH}_{2}\right)_{4}, 2 \times \mathrm{OH}\right], 1.46(8 \mathrm{H}, \mathrm{q}, J=7.5 \mathrm{~Hz}$, $\left.4 \times \mathrm{CH}_{2}\right) ; \delta_{\mathrm{C}} 7.7\left(\mathrm{CH}_{3}\right), 24.0,31.0,38.1\left(\mathrm{CH}_{2}\right), 74.6(\mathrm{COH}) ; m / z 194\left(\mathrm{M}^{+}-2 \mathrm{H}_{2} \mathrm{O}, 1 \%\right), 183(13)$, 165 (65), 109 (23), 97 (43), 95 (20), 87 (100), 85 (24), 69 (38), 57 (77), 55 (21).

3,9-Diethylundecane-3,9-diol (6k). ${ }^{19}$ White solid; mp $82-83{ }^{\circ} \mathrm{C}$ (dichloromethane/hexane); $R_{\mathrm{f}}$ 0.27 (hexane/ethyl acetate: $2 / 1) ; v(\mathrm{KBr}) 3450-3210(\mathrm{OH}), 2968,2933,2878,1466 \mathrm{~cm}^{-1} ; \delta_{\mathrm{H}} 0.85$ $\left(12 \mathrm{H}, \mathrm{t}, J=7.5 \mathrm{~Hz}, 4 \times \mathrm{CH}_{3}\right), 1.25-1.42\left[12 \mathrm{H}, \mathrm{m},\left(\mathrm{CH}_{2}\right)_{5}, 2 \times \mathrm{OH}\right], 1.45(8 \mathrm{H}, \mathrm{q}, J=7.5 \mathrm{~Hz}$, $\left.4 \times \mathrm{CH}_{2}\right) ; \delta_{\mathrm{C}} 7.7\left(\mathrm{CH}_{3}\right), 23.3,30.9,30.95,38.1\left(\mathrm{CH}_{2}\right), 74.5(\mathrm{COH}) ; m / z 208\left(\mathrm{M}^{+}-2 \mathrm{H}_{2} \mathrm{O}, 2 \%\right), 197$ (17), 179 (64), 123 (21), 111 (42), 109 (28), 97 (20), 95 (28), 87 (100), 85 (31), 69 (45), 57 (90), 55 (26). 
1,8-Diphenyloctane-1,8-diol (61). ${ }^{20}$ Diastereomeric mixture: White solid; $\mathrm{mp} \quad 83-84^{\circ} \mathrm{C}$ (dichloromethane/hexane); $R_{f} 0.30$ (hexane/ethyl acetate: $2 / 1$ ); $v(\mathrm{KBr}) 3520-3180(\mathrm{OH}), 3083$, $3058,3022 \mathrm{~cm}^{-1}(\mathrm{ArH}) ; \delta_{\mathrm{H}} 1.19-1.69\left(14 \mathrm{H}, \mathrm{m}, 6 \times \mathrm{CH}_{2}, 2 \times \mathrm{OH}\right), 4.54(2 \mathrm{H}, \mathrm{dd}, J=7.2,6.1$, $2 \times \mathrm{CHOH}), 7.21-7.29(10 \mathrm{H}, \mathrm{m}, 2 \times \mathrm{ArH}) ; \delta_{\mathrm{C}} 25.1,28.8,38.4\left(\mathrm{CH}_{2}\right), 73.9(\mathrm{CHOH}), 125.3,126.8$, 127.8, 144.3 (ArC); m/z $280\left(\mathrm{M}^{+}-\mathrm{H}_{2} \mathrm{O}, 2\right.$ \%), 207 (37), 174 (64), 158 (15), 117 (52), 107 (100), 105 (26), 104 (83), 91 (30), 79 (61), 77 (36).

Double lithiation of compounds 1 followed by reaction with carbonyl compounds as electrophiles (Grignard-type reaction conditions, Method B). Preparation of diols 6

Isolation of compound 6. Method B: General procedure. To a blue suspension of lithium powder $(0.070 \mathrm{~g}, 10 \mathrm{mmol})$ and a catalytic amount of DTBB $(0.027 \mathrm{~g}, 0.1 \mathrm{mmol})$ in THF $(3 \mathrm{~mL})$ was added the corresponding 1,n-bis(phenylsulfanyl)alkane $1(1.0 \mathrm{mmol})$ at $-78^{\circ} \mathrm{C}$. The reaction mixture was stirred for $1 \mathrm{~h}$ at the same temperature and after that, the corresponding carbonyl compound $(2.2 \mathrm{mmol})$ was added dropwise and after $15 \mathrm{~min}$ it was hydrolyzed with water (4 $\mathrm{mL})$ and extracted with ethyl acetate $(3 \times 10 \mathrm{~mL})$. The organic layer was dried over anhydrous magnesium sulfate and evaporated (15 Torr). The residue was purified by column chromatography (silica gel; hexane/ethyl acetate) to yield pure products 6 . Yields and structures are included in Table 1. Physical and spectroscopic data as well as literature references follow.

2,2,8,8-Tetramethylnonane-3,7-diol (6b). Diastereomeric mixture: White solid; mp 77-85 ${ }^{\circ} \mathrm{C}$ (dichloromethane/hexane); $R_{\mathrm{f}} 0.31$ (hexane/ethyl acetate: 2/1); $v(\mathrm{KBr}) 3550-3210(\mathrm{OH}), 2969$, $2869,1475,1392,1373 \mathrm{~cm}^{-1} ; \delta_{\mathrm{H}} 0.89\left[18 \mathrm{H}, \mathrm{s}, 2 \times\left(\mathrm{CH}_{3}\right)_{3} \mathrm{C}\right], 1.29-1.37\left(2 \mathrm{H}, \mathrm{m}, \mathrm{CH}_{2}\right), 1.52-1.67$ $\left(6 \mathrm{H}, \mathrm{m}, 2 \times \mathrm{CH}_{2}, 2 \times \mathrm{OH}\right), 3.18-3.23(2 \mathrm{H}, \mathrm{m}, 2 \times \mathrm{CHOH}) ; \delta_{\mathrm{C}} 24.5\left(\mathrm{CH}_{2}\right), 25.6\left(\mathrm{CH}_{3}\right), 31.0,31.6$, 34.9, $34.95\left(\mathrm{CH}_{2}\right), 79.8,80.1(\mathrm{CHOH}) ; m / z 198\left(\mathrm{M}^{+}-\mathrm{H}_{2} \mathrm{O}, 2 \%\right), 141$ (12), 123 (77), 97 (20), 95 (15), 85 (20), 83 (22), 81 (39), 71 (18), 69 (34), 67 (18), 59 (18), 57 (100), 55 (53), 43 (67), 41 (74); HRMS: $\mathrm{M}^{+}-\mathrm{H}_{2} \mathrm{O}$, found 198.1987. $\mathrm{C}_{13} \mathrm{H}_{26} \mathrm{O}$ requires 198.1984 .

1,7-Diphenylheptane-2,6-diol (6d). Diastereomeric mixture: Pale yellow oil; $R_{\mathrm{f}} 0.14$ (hexane/ethyl acetate: 2/1); $v$ (film) 3520-3170 (OH), 3060, 3034, 2982, 2941, 2863, $1455 \mathrm{~cm}^{-1}$; $\delta_{\mathrm{H}} 1.45-1.57\left[6 \mathrm{H}, \mathrm{m},\left(\mathrm{CH}_{2}\right)_{3}\right], 1.82(2 \mathrm{H}$, br s, $2 \times \mathrm{OH}), 2.64(2 \mathrm{H}, \mathrm{dd}, J=13.8,8.2 \mathrm{~Hz}, 2 \times \mathrm{PhCH})$, 2.77-2.82 (2H, m, $2 \times \mathrm{PhCH}), 3.77-3.82(2 \mathrm{H}, \mathrm{m}, 2 \times \mathrm{CHOH}), 7.18-7.31(10 \mathrm{H}, \mathrm{m}, \mathrm{ArH}) ; \delta_{\mathrm{C}} 21.7$, 21.9, 36.4, 36.5, 44.0, $44.1\left(\mathrm{CH}_{2}\right), 72.4,72.5(\mathrm{CHOH}), 126.4,128.45,128.5,129.4,138.0 ; \mathrm{m} / z$ $284\left(\mathrm{M}^{+}, 3 \%\right), 175$ (18), 130 (24), 129 (17), 92 (20), 91 (100), 77 (15), 65 (20), 44 (30); HRMS: $\mathrm{M}^{+}$, found 284.1778. $\mathrm{C}_{19} \mathrm{H}_{24} \mathrm{O}_{2}$ requires 284.1776 .

3,7-Diethylnonane-3,7-diol (6e). White solid; mp 84-86 ${ }^{\circ} \mathrm{C}$ (dichloromethane/hexane) (found: C, 72.02; H, 13.12. $\mathrm{C}_{13} \mathrm{H}_{28} \mathrm{O}_{2}$ requires: $\left.\mathrm{C}, 72.17 ; \mathrm{H}, 13.04\right) ; R_{\mathrm{f}} 0.22$ (hexane/ethyl acetate: $2 / 1$ ); $v$ (KBr) $3540-3210(\mathrm{OH}), 2951,2917,2849,1470 \mathrm{~cm}^{-1} ; \delta_{\mathrm{H}} 0.78\left(12 \mathrm{H}, \mathrm{t}, J=7.4 \mathrm{~Hz}, 4 \times \mathrm{CH}_{3}\right), 1.18-$ $1.37\left[8 \mathrm{H}, \mathrm{m},\left(\mathrm{CH}_{2}\right)_{3}, 2 \times \mathrm{OH}\right], 1.40\left(8 \mathrm{H}, \mathrm{q}, J=7.4 \mathrm{~Hz}, 4 \times \mathrm{CH}_{2}\right) ; \delta_{\mathrm{C}} 7.7\left(\mathrm{CH}_{3}\right), 17.1,31.0,38.7$ $\left(\mathrm{CH}_{2}\right), 74.5(\mathrm{COH}) ; \mathrm{m} / z 180\left(\mathrm{M}^{+}-2 \mathrm{H}_{2} \mathrm{O}, 5 \%\right), 151$ (45), 109 (21), 97 (26), 96 (54), 95 (24), 87 (25), 81 (36), 69 (25), 67 (42), 57 (50), 55 (100), 43 (33), 41 (54).

1-[3-(1-Hydroxycyclohexyl)propyl]cyclohexanol (6f). ${ }^{21}$ White solid; mp $121-122{ }^{\circ} \mathrm{C}$ (dichloromethane/hexane); $R_{\mathrm{f}} 0.17$ (hexane/ethyl acetate: $\left.2 / 1\right) ; v(\mathrm{KBr}) 3450-3220(\mathrm{OH}), 2934$, $1445 \mathrm{~cm}^{-1} ; \delta_{\mathrm{H}} 1.24-1.64\left(26 \mathrm{H}, \mathrm{m}, 13 \times \mathrm{CH}_{2}\right), 2.05(2 \mathrm{H}$, br s, $2 \times \mathrm{OH}) ; \delta_{\mathrm{C}} 22.2,22.3,25.8,37.5$, 
$42.8\left(\mathrm{CH}_{2}\right), 71.5(\mathrm{COH}) ; m / z 222\left(\mathrm{M}^{+}-\mathrm{H}_{2} \mathrm{O}, 2 \%\right), 127$ (11), 121 (14), 109 (26), 108 (80), 99 (40), 96 (19), 93 (36), 81 (60), 79 (71), 67 (100), 54 (91), 43 (41), 41 (86).

1-[3-(1-Hydroxycyclooctyl)propyl]cyclooctanol $\quad(\mathbf{6 g}) .^{21}$ White solid; mp 144-145 ${ }^{\circ} \mathrm{C}$ (dichloromethane/hexane); $R_{\mathrm{f}} 0.15$ (hexane/ethyl acetate: $\left.2 / 1\right) ; v(\mathrm{KBr}) 3520-3250(\mathrm{OH}), 2951$, $2917,2848,1470 \mathrm{~cm}^{-1} ; \delta_{\mathrm{H}} 1.36-1.70\left(36 \mathrm{H}, \mathrm{m}, 17 \times \mathrm{CH}_{2}, 2 \times \mathrm{OH}\right) ; \delta_{\mathrm{C}} 16.6,22.3,25.0,28.2,36.3$, $42.0\left(\mathrm{CH}_{2}\right), 74.9(\mathrm{COH}) ; m / z 260\left(\mathrm{M}^{+}-2 \mathrm{H}_{2} \mathrm{O}, 11 \%\right), 149$ (28), $136(74), 121$ (41), 108 (62), 107 (33), 95 (71), 94 (50), 93 (33), 91 (26), 81 (82), 80 (45), 79 (53), 67 (78), 55 (67), 43 (81), 41 (100).

(1S,2S,5R,1'S,2'S,5'R)-1-[3-(1'-Hydroxy-2'-isopropyl-5'-methylcyclohexyl)propyl]-2isopropyl-5-methylcyclohexanol (6h). Colourless oil; $R_{\mathrm{f}} 0.71$ (hexane/ethyl acetate: $2 / 1$ ); $v$ (film) 3630-3340 (OH), 2952, 2868, 1464, $1373 \mathrm{~cm}^{-1} ; \delta_{\mathrm{H}} 0.82-1.78(42 \mathrm{H}, \mathrm{m}), 2.06-2.11(2 \mathrm{H}, \mathrm{m})$; $\delta_{\mathrm{C}} 18.0\left(\mathrm{CH}_{2}\right), 18.15\left(\mathrm{CH}_{3}\right), 20.5\left(\mathrm{CH}_{2}\right), 22.4,23.6\left(\mathrm{CH}_{3}\right), 25.5,28.0(\mathrm{CH}), 35.1,41.9,46.7$ $\left(\mathrm{CH}_{2}\right), 48.1(\mathrm{CH}), 75.0(\mathrm{COH}) ; \mathrm{m} / z 334\left(\mathrm{M}^{+}-\mathrm{H}_{2} \mathrm{O}, 1 \%\right), 165$ (15), 164 (100), 163 (18), 149 (26), 137 (25), 135 (35), 121 (27), 109 (38), 107 (17), 95 (53), 93 (26), 83 (17), 81 (58), 69 (48), 67 (34), 55 (56), 43 (56), 41 (58); HRMS: $\mathrm{M}^{+}-\mathrm{H}_{2} \mathrm{O}$, found 334.3233. $\mathrm{C}_{23} \mathrm{H}_{42} \mathrm{O}$ requires 334.3236. $[\alpha]_{\mathrm{D}}{ }^{20}=+4.2^{\circ}$ (c 1.07, dichloromethane).

1,1,5,5-Tetraphenylpentane-1,5-diol (6i). Yellow oil; $R_{\mathrm{f}} 0.57$ (hexane/ethyl acetate: 2/1); $v$ (film) 3510-3140 (OH), 3060, 3030, 2940, 2860, $1460 \mathrm{~cm}^{-1} ; \delta_{\mathrm{H}} 1.30-1.39\left(2 \mathrm{H}, \mathrm{m}, \mathrm{CH}_{2} \mathrm{CH}_{2} \mathrm{CH}_{2}\right)$, $2.18(1 \mathrm{H}$, br s, $2 \times \mathrm{OH}), 2.18-2.31\left(4 \mathrm{H}, \mathrm{m}, 2 \times \mathrm{CH}_{2} \mathrm{CH}\right), 7.17-7.34(20 \mathrm{H}, \mathrm{m}, \mathrm{ArH}) ; \delta_{\mathrm{C}} 18.3,42.0$ $\left(\mathrm{CH}_{2}\right), 78.3(\mathrm{COH}), 125.9,126.7,128.1146 .9$ (ArC); $m / z 372\left(\mathrm{M}^{+}-2 \mathrm{H}_{2} \mathrm{O}, 20 \%\right), 352(20), 281$ (30), 215 (21), 202 (19), 193 (36), 191 (29), 179 (27), 165 (16), 115 (21), 91 (37), 43 (100); HRMS: $\mathrm{M}^{+}-2 \mathrm{H}_{2} \mathrm{O}$, found 372.1883. $\mathrm{C}_{29} \mathrm{H}_{24}$ requires 372.1878 .

\section{Acknowledgements}

This work was generously supported by the Spanish Ministerio de Educación y Ciencia (MEC; grant no. CTQ2004-01261) and the Generalitat Valenciana (GV; grants no. GRUPOS05/052 and GRUPOS05/058). A.A. thanks the University of Alicante for a predoctoral fellowship. We also thank MEDALCHEMY S.L. for a gift of chemicals, especially lithium powder.

\section{References and Footnotes}

1. See, for instance: Corey, E. J.; Seebach, D. J. Org. Chem. 1966, 31, 4097.

2. (a) Block, E. In Reactions of Organosulphur Compounds; Academic Press: New York, 1978; p 42. (b) Krief, A. Tetrahedron 1980, 36, 2531.

3. (a) Corey, E. J.; Seebach, D. Angew. Chem. Int. Ed. Engl. 1965, 4, 1075. (b) Corey, E. J.; Seebach, D. Angew. Chem. Int. Ed. 1965, 4, 1077. (c) Seebach, D.; Corey, E. J. J. Org. Chem. 1975, 40, 231. (d) For a review on acyl main group metal and metalloid derivatives, see: Nájera, C.; Yus, M. Org. Prep. Proced. Int. 1995, 27, 383. 
4. (a) Screttas, C. G.; Micha-Screttas, M. J. Org. Chem. 1978, 43, 1064. (b) Screttas, C. G.; Micha-Screttas, M. J. Org. Chem. 1979, 44, 713. (c) For a review, see: Cohen, T.; Bhupathy, M. Acc. Chem. Res. 1982, 22, 152. (d) For a recent application of this stoichiometric lithiation reaction, see: Deng, K.; Bensari-Bouguerra, A.; Whetstone, J.; Cohen, T. J. Org. Chem. 2006, 71, 2360.

5. (a) Yus, M. Chem. Soc. Rev. 1996, 155. Last papers from our laboratory using this methodology: (b) Meléndez, J.; Alonso, F.; Yus, M. Tetrahedron Lett. 2006, 47, 1187. (c) Alonso, F.; Meléndez, J.; Soler, T.; Yus, M. Tetrahedron 2006, 62, 2264. (d) Alonso, F.; Meléndez, J.; Yus, M. Tetrahedron 2006, 62, 4814.

6. For reviews, see: (a) Foubelo, F.; Yus, M. Trends Org. Chem. 1998, 7, 1. (b) Foubelo, F.; Yus, M. Curr. Org. Chem. 2005, 9, 459.

7. For monographs, see: (a) Wakefield, B. J. Organolithium Methods; Academic Press: London, 1988. (b) Sapse, A. M.; von Ragué Schleyer, P., Eds. Lithium Chemistry: A Theoretical and Experimental Overview; J. Wiley \& Sons: New York, 1995. (c) Gray, M.; Tinkel, M.; Snieckus, V. In Comprehensive Organometallic Chemistry II; Abel, E. W.; Stone, F. G. A.; Wilkinson, G.; McKillop, A., Eds.; Pergamon: Oxford, 1995; Vol. 11, pp 192. (d) Clayden, J. Organolithiums: Selectivity for Synthesis; Pergamon: Oxford, 2002. For a review on metal-promoted dehalogenation, see: (e) Alonso, F.; Beletskaya, I. P.; Yus, M. Chem. Rev. 2002, 102, 4009.

8. (a) Meyers, A. I.; Licini, G. Tetrahedron Lett. 1989, 30, 4049. (b) Negishi, E.-I.; Swanson, D. R.; Rousset, C. J. J. Org. Chem. 1990, 55, 5406. (c) Weber, B.; Seebach, D. Tetrahedron 1994, 50, 7473.

9. For a preliminary communication, see: Foubelo, F.; Yus, M. Tetrahedron Lett. 2000, 41, 5047.

10. See, for instance: Almena, J.; Foubelo, F.; Yus, M. Tetrahedron 1995, 51, 11883.

11. Miranda-Soto, V.; Parra-Hake, M.; Morales-Morales, D.; Toscazo, R. A.; Boldt, G.; Koch, A.; Grotjahn, D. B. Organometallics 2005, 24, 5569.

12. Cattalini, L.; Martelli, M. J. Am. Chem. Soc. 1969, 91, 312.

13. Clark, J. H.; Miller, J. M. J. Chem. Soc., Chem. Commun. 1976, 229.

14. Cronin, J. P.; Dilworth, B. M.; McKervey, M. A. Tetrahedron Lett. 1986, 27, 757.

15. Russell, G. A.; Ngoviwatchai, P.; Tashtoush, H. I.; Pla-Dalmau, A.; Khanna, R. K. J. Am. Chem. Soc. 1988, 110, 3530.

16. Rao, S. A.; Periasamy, M. Tetrahedron Lett. 1988, 29, 1583.

17. Zhang, H.-C.; Harris, B. D.; Costanzo, M. J.; Lawson, E. C.; Maryanoff, C. A.; Maryanoff, B. E. J. Org. Chem. 1998, 63, 7964.

18. Colonge, J.; Poilane, G. Bull. Soc. Chim. Fr. 1955, 499.

19. Abou, A.; Foubelo, F.; Yus, M. Tetrahedron 2006, 62, 10417.

20. Foubelo, F.; Abou, A.; Yus, M. Eur. J. Org. Chem. 2005, 5089.

21. Kostas, I. D.; Screttas, C. G. J. Org. Chem. 1997, 62, 5575. 